\title{
Kommt in der evidenzbasierten Medizin die Patientensicht zu kurz?
}

Soll der Einsatz von Phytotherapeutika an erster Stelle an wissenschaftlichen Daten zu einzelnen Präparaten ausgerichtet werden, wie es in der evidenzbasierten Medizin (EBM) üblich ist, oder sollen $\mathrm{Pa}$ tientenwünsche und positive praktische Erfahrungen mit Naturheilmitteln Vorrang haben? Beim Debattengipfel „Naturmedizin 2012" in Hamburg wurde diese Frage lebhaft diskutiert.

Vor der Debatte votierte die deutliche Mehrheit des Plenums für die These, dass die Patientenwirklichkeit bei der Naturmedizin von höchster Bedeutung sei. Nach den Impulsvorträgen von je drei Experten für jede Seite könnte die Zahl der EBM-Kritiker bei Naturheilmitteln sogar noch etwas gewachsen sein. Dabei hatten beide Seiten gute Argumente vorgebracht, so gute, dass auch die Frage berechtigt ist, ob EBM und Patientenwirklichkeit absolut ein „Entweder - Oder“ sein müssen oder nicht auch gut miteinander auskommen können.
Evidenz ist nötig für eine individualisierte Medizin, betonte Professor Gerd Glaeske, Bremen, Debattenführer der einen Fraktion. Phytotherapie sei keine besondere Richtung der Pharmakotherapie, sondern beruhe ebenfalls auf Naturwissenschaften und sollte rational, also wissenschaftlich begründbar, angewendet werden. Dem entgegnete Professor Michael Habs, Geschäftsführer bei Dr. Willmar Schwabe und anderer Debattenführer: „Erfolg zeigt sich an dem, was mit dem Individuum passiert (...) Man muss für die vorhandene Medizin den richtigen Patienten finden."

Natürlich müssen Extrakte als Voraussetzung für evidenzbasierte Phytotherapie analysiert, klinisch getestet und reproduzierbar sein, wie Professor Theodor Dingermann, Frankfurt/Main, betonte. Aber sollten nur randomisierte klinische Studien zu einem Extrakt als Wirksamkeitsbeleg gelten? Naturheilmittel modifizieren in günstiger Weise die Erwar- tungshaltung des Patienten, weil sie hoch akzeptiert sind und wenig Nebenwirkungen haben, sagte Professor Manfred Schablowski, Essen. Dies könnte die Wirksamkeit der Phytotherapie verstärken.

Die Gegenseite konterte: „Natur ist nicht von Natur aus gut" und kritisierte den „Mythos der Nebenwirkungsfreiheit“ von Phytotherapeutika.

Besonders emotional dozierte Dr. Berthold Musselmann, Heidelberg: „Medizin ist eine Erfahrungswissenschaft", betonte er, die rein wissenschaftliche Medizin sei ein Irrweg. Und fragte ins Plenum: „Warum wenden wohl so viele Ärzte, auch hartgesottene Schulmediziner, bei sich selbst Naturheilmittel an?"

Roland Fath, freier Medizinjournalist

Debattengipfel Naturmedizin 2012,

Hamburg, 24.10. 2012

Veranstalter: Dr. Willmar Schwabe

\section{Gute evidenzbasierte Datenlage für die Add-on-Therapie in der refraktären Situation}

Die Revision des internationalen Diagnosemanuals DSM hat zur Diskussion über die generalisierte Angststörung (GAD) als eigene Entität geführt. Jetzt ist klar: Die Diagnose bleibt mit kleinen Veränderungen erhalten und der neue ICD-Katalog wird sich dem weitgehend anschließen. Damit kann wieder die evidenzbasierte Therapie der GAD in den Mittelpunkt des Interesses rücken. Sie erfolgt nach internationalen Leitlinien vorzugsweise mit Selektiven Serotonin-Wiederaufnahmehemmern (SSRI), SerotoninNoradrenalin-Wiederaufnahmehemmern (SNRI) oder Pregabalin (Lyrica ${ }^{\circledast}$ ) [Bandelow B et al. World J Bio Psychiatry 2008; 9:248]. Allerdings sind die Rückfallraten in Studien hoch. Eine Umfrage unter Ärzten ergab laut Bandelow, dass die meisten in einem solchen Falle dazu tendieren, die Dosis des Erstlinienpräparats $\mathrm{zu}$ erhöhen. Evidenz gibt es dafür ebenso wenig wie für den Wechsel auf ein anderes Medikament, so Bandelow. Auch Add-on-Strategien mit atypischen Antipsychotika konnten in Studien häufig nicht ihre Wirksamkeit beweisen.

Die beste Datenlage besteht für eine Add-on-Therapie mit Pregabalin, so Bandelow. Eine Studie untersuchte Wirksamkeit und Sicherheit von Pregabalin in einer Dosis von 150-600 mg/d versus Placebo zusätzlich zu Escitalopram, Paroxetin oder Venafaxin, nachdem diese Wirkstoffe bei 356 Patienten nicht zu einem ausreichenden Therapieerfolg ( $\mathrm{Ha}$ milton-Angst-Skala (HAM-A)-Wert < 16) geführt hatten [Rickels $K$. et al. Int Clin Psychopharmacol 2012; 27 (3): 142 150]. Betrugen die entsprechenden Werte zu Beginn des Interventionszeitraums 20,7 in der Pregabalin- und 21,4 in der Placebogruppe, sank der HAM-A-Wert bei aktiver Add-on-Therapie um 7,6
Punkte und damit signifikant stärker als bei Placebogabe $(-6,4 ; p<0,05)$. Bei $47,5 \%$ der Patienten in der PregabalinGruppe verringerten sich die Angstsymptome nach dem HAM-A um die Hälfte und mehr, bei Placeboeinnahme nur bei $35,2 \%(p=0,0145)$. Unter der Add-onTherapie waren Nebenwirkungen wie Schwindel, Kopfschmerzen oder Somnolenz etwas häufiger als in der Kontrollgruppe. Bandelow betonte, dies sei die einzige Studie mit einer adäquaten Teststärke, die in der refraktären Situation einen Erfolg der Add-on-Therapie gezeigt habe.

Friederike Klein, freie Medizinjournalistin

Satellitensymposium „Psychiater, übernehmen Sie - wann ist der Facharzt bei GAD gefragt", DGPPN-Kongress 2012, Berlin, 22.11.2012

Veranstalter Pfizer 